\title{
ON STRONGLY PRIME RINGS AND IDEALS
}

\author{
ALGIRDAS KAUČIKAS AND ROBERT WISBAUER
}

\begin{abstract}
Strongly prime rings may be defined as prime rings with simple central closure. This paper is concerned with further investigation of such rings. Various characterizations, particularly in terms of symmetric zero divisors, are given. We prove that the central closure of a strongly (semi-)prime ring may be obtained by a certain symmetric perfect one sided localization. Complements of strongly prime ideals are described in terms of strongly multiplicative sets of rings. Moreover, some relations between a ring and its multiplication ring are examined.
\end{abstract}

\section{TeRminology And BASIC RESUlts}

All rings in this paper are associative with identity element which should be preserved by ring homomorphisms, and $R$-Mod denotes the category of unital left modules over the ring $R$. By an ideal of the ring we shall understand a two-sided ideal. We denote by $\left\{a_{1}, \ldots, a_{n}\right\}$ the set consisting of the elements $a_{1}, \ldots, a_{n} \in R$, and by $\left(a_{1}, \ldots, a_{n}\right)$ the ideal of the ring generated by these elements. Particularly, $(a)$ denotes the ideal generated by the element $a \in R$. $A \subset B$ means that $A$ is proper subset of $B$.

Let $R$ be an algebra over a commutative ring $\Lambda$, and $R^{\circ}$ its opposite ring. Then the enveloping algebra $R^{e}=R \otimes_{\Lambda} R^{\circ}$ acts canonically on $R$ from the left.

The quotient ring $R^{e} / A n n_{R^{e}} R$ is called the multiplication ring of $R$ and will be denoted by $M(R)$. Easy arguments show that the definition of $M(R)$ does not depend of the ring $\Lambda$. Thus $R$ is a faithful left $M(R)$-module, its ideals are exactly the $M(R)$-submodules and $\operatorname{End}_{M(R)} R$ is isomorphic to the centre of $R$, which we denote by $Z(R)$.

$M(R)$ may be equivalently defined as the subring of $E n d_{\Lambda} R$, acting from the left on $R$, generated as a ring by all left and right mutiplications $l_{a}$ and $r_{b}$, where $a, b \in R$, and $l_{a} x=a x, r_{b} x=x b$, for $x \in R$. So each $\lambda \in M(R)$ is of the form $\lambda=\sum_{k} l_{a_{k}} r_{b_{k}}$, where $a_{k}, b_{k} \in R$, and can be represented as the sum $\sum_{k} a_{k} \otimes b_{k}^{\circ}$, where $b_{k}^{\circ} \in R^{\circ}$. Then $\lambda x=\sum_{k} a_{k} x b_{k}, x \in R$. It's clear that the canonical embedding $R \hookrightarrow M(R)$, sending $a \in R$ to $l_{a}$ is onto if and only if $R$ is commutative.

The map $\pi: M(R) \rightarrow R, \lambda \mapsto \lambda 1$, is an $M(R)$-module homomorphism.

The research of the first author was supported by DAAD. 
If $R$ is a central simple algebra over the field $F$, its multiplication ring is isomorphic to $R \otimes_{F} R^{\circ}$ which is also central simple over $F$ (see [3], Ch. 3, Prop. 4.2). If $R$ is an Azumaya algebra then there are canonical isomorphisms $M(R) \cong R \otimes_{Z(R)} R^{\circ} \cong \operatorname{End}_{Z(R)} R$.

Let $M$ be an $R$-bimodule, and $Z_{M}=Z_{M}(R)=\{\delta \in M \mid r \delta=\delta r, r \in R\}$ the set of $R$-centralizing elements of $M$. If $M=R Z_{M}$ then $M$ is called a centred R-bimodule.

Let $\phi: R \rightarrow S$ be a ring homomorphism. Then $S$ becomes a canonical $R$-bimodule. We call $\phi$ a centred homomorphism, and $S$ a centred extension of $R$ (via $\phi$ ), provided $S$ is a centred $R$-bimodule under this structure. Of course, $Z_{S}=Z_{S}(R)$ is a subring of $S$. It easily follows from the definition that each centred extension of the ring $R$ can be obtained as a factor ring of a semigroup ring $R[G]$, where $G$ is a free semigroup with unit. Rings and their centred homomophisms form a category (called Procesi category).

For a semiprime ring $R$ we denote by $Q(R)$ the central closure and by $F(R)$ the extended centroid of the ring $R$. By definition, $F(R)$ is the centre of $Q(R)$ and is a field when $R$ is a prime ring. See [5], [19] for definitions and basic properties of these rings.

\section{Strongly PRIME RINGS}

Let $M$ be left $R$-module. We say that $N \in R$-Mod is subgenerated by $M$ if $N$ is a submodule of an $M$-generated module (see the [19]). The category of $M$-subgenerated modules is denoted by $\sigma[M]$.

A nonzero $R$-module $M$ is called strongly prime if it is subgenerated by each of its nonzero submodules. In terms of elements, $M \in R$-Mod is strongly prime if and only if for any non-zero $x, y \in M$, there exits finite set of elements $\left\{a_{1}, \ldots, a_{n}\right\} \subseteq R, \quad n=n(x, y)$, such that $A n n_{R}\left\{a_{1} x, \ldots, a_{n} x\right\} \subseteq A n n_{R}\{y\}$ (see [4]). Other characterizations and properties of strongly prime modules can be found in [4], [19].

Taking $M=R$ in the definition of strongly prime modules over $R$, the notion of left strongly prime ring is obtained (see [9]).

We look at a ring $R$ as an $R$-bimodule and consider $R$ as the left module over its multiplication ring $M(R)$. Now $R$ is called strongly prime if $R$ is a strongly prime module over $M(R)$.

We say an element $a \in R$ is a symmetric zero divisor if for any finite subset of elements $\left\{a_{1}, \ldots, a_{n}\right\} \subseteq(a), A n n_{M(R)}\left\{a_{1}, \ldots, a_{n}\right\} \nsubseteq A n n_{M(R)}\left\{1_{R}\right\}$. Denote by $z d(R)$ the set of symmetric zero divisors of $R$.

Of course, for $R$ commutative, taking $n=1$ and $a_{1}=a$, we obtain the usual definition of zero divisors. 
Theorem 2.1. For any ring $R$ the following are equivalent:

(1) $R$ is a strongly prime ring;

(2) $z d(R)=0$;

(3) $R$ is a prime ring and the central closure $Q(R)$ of the ring $R$ is a simple ring;

(4) for any nonzero $a, b \in R$, there exist $\lambda_{1}, \ldots, \lambda_{n} \in M(R)$ such that $A n n_{M(R)}\left\{\lambda_{1} a, \ldots, \lambda_{n} a\right\} \subseteq A n n_{M(R)}\{b\} ;$

(5) for any nonzero $a \in R$, there exist $\lambda_{1}, \ldots, \lambda_{n} \in M(R)$ such that $\operatorname{Ann}_{M(R)}\left\{\lambda_{1} a, \ldots, \lambda_{n} a\right\} \subseteq A n n_{M(R)}\left\{1_{R}\right\} ;$

$\left(5^{\prime}\right)$ for any nonzero $a \in R$, there exist $a_{1}, \ldots, a_{n} \in(a)$, such that

$$
\sum_{i} x_{i} a_{k} y_{i}=0, \text { for all } 1 \leq k \leq n \text {, implies } \sum_{i} x_{i} y_{i}=0 ;
$$

(6) there exists a centred monomorphism $\phi: R \rightarrow K$ where $K$ is a simple ring;

(7) there exists a centred monomorphism $\phi: R \rightarrow S$, where the ring $S$ has the following property: for each nonzero ideal $I \subseteq R$, its extension $I^{\varepsilon}$ in $S, I^{\varepsilon}=S I S$, is equal to $S$.

Proof. The equivalence of conditions (1), (3), (4), (5) is proved in [19], Theorem 35.6. Obviously, $(3) \Rightarrow(6) \Rightarrow(7)$.

We prove $(7) \Rightarrow(5)$. Take any nonzero $a \in R$. Then, by assumption, $(a)^{\varepsilon}=(a) Z_{S}=S$. This gives an expression $\sum_{k} a_{k} \delta_{k}=1$, with $a_{k} \in(a)$, $\delta_{k} \in Z_{S}$. So we obtain $A n n_{M(R)}\left\{a_{1}, \ldots, a_{n}\right\} \subseteq A n n_{M(R)}\left\{1_{R}\right\}$.

The equivalence of (2) and (5) easily follows from the definition of symmetric zero divisors.

$\left(5^{\prime}\right)$ is exactly (5) written in terms of elements of the ring $R$.

Particularly by (2) of this theorem, each ring which is not strongly prime has nonzero symmetric zero divisors. It is also clear that a strongly prime ring is left and right strongly prime in the sense of Handelman-Lawrence.

We note that for any strongly prime ring $R$, the central closure $Q(R)$ coincides with the right (and left) Martindale ring of quotients $Q_{r}(R)$, and so with the symmetric ring of quotients (see [12, Proposition 1.2, 1.4]): Indeed, let any element of $Q_{r}(R)$ be represented by the homomorphism of right $R$-modules $f: I \rightarrow R$, where $I \subseteq R$ is a nonzero ideal. $Q(R)$ is a simple ring so we obtain an expression

$$
\sum_{k} i_{k} u_{k}=1 \text {, with } i_{k} \in I, u_{k} \in F(R) \text {. }
$$

Multiplying this equality by $f$ from the left, we obtain $f \in Q(R)$, because $f i_{k} \in R$. 
Let $M$ be a centred $R$-bimodule, $\delta \in Z_{M}$ - an $R$-centralizing element. For an ideal $I \subseteq R$ consider the ideals

$$
I_{1}=I, I_{k+1}=\left\{r \in R \quad \mid r \delta \in I_{k} M\right\}, \quad k \in \mathbb{N} .
$$

Obviously, $I_{k} \subseteq I_{k+1}$ for all $k \in \mathbb{N}$, and, because $M$ is a centred bimodule, $I_{k} M=I_{k} Z_{M}$, for all $k \in \mathbb{N}$.

This construction yields a further characterization of strongly prime rings.

Theorem 2.2. A ring $R$ is strongly prime if and only if there exits a centred $R$-bimodule $M$ and an element $\delta \in Z_{M}$, with $A n n_{R} \delta=0$ and having the property: for each nonzero ideal $I \subseteq R, I_{n+1}=R$ for some $n \in \mathbb{N}$.

Proof. If $R$ is strongly prime, then we may take $M=Q(R)$ - the central closure of $R$ - and $\delta=1$. Then $I Q(R)=Q(R)$ and $I_{2}=R$, for each nonzero ideal $I \subseteq R$.

Let some centred $R$-bimodule $M$ and $\delta \in Z_{M}$ satify the condition of the theorem. Take any nonzero $a \in R$ and let $I=(a)$. So

$$
\delta=a_{1}^{(n)} \delta_{1}^{(n)}+\ldots+a_{m}^{(n)} \delta_{m}^{(n)}, \text { with some } a_{i}^{(n)} \in I_{n}, \quad \delta_{i}^{(n)} \in Z_{M} .
$$

By definition of the ideals $I_{k}$, we obtain finite linear combinations

$$
a_{i}^{(n)} \delta=\sum_{j} a_{j}^{(n-1)} \delta_{j}^{(n-1)}, \quad a_{i}^{(k+1)} \delta=\sum_{j} a_{j}^{(k)} \delta_{j}^{(k)},
$$

with $a_{j}^{(k)} \in I_{k}$ and $\delta_{j}^{(k)} \in Z_{M}, 1 \leq k \leq n-1$.

Particularly, $a_{i}^{(1)} \in(a)$. Now, if $\lambda a_{i}^{(1)}=0$ for some $\lambda \in M(R)$, and all $i$, then all $\lambda a_{i}^{(2)} \delta=0$, and step by step we obtain that all $\lambda a_{i}^{(k)} \delta=0, \quad 1 \leq k \leq n$, and $\lambda 1 \delta=0$, so $\lambda 1=0$ because $A n n_{R} \delta=0$. By (5) of Theorem 2.1, $R$ is strongly prime.

The central closure $Q(R)$ of any strongly prime ring $R$ has an important universal property. In [11] the following result was proved. Let the ring $R$ be centrally embedded into a ring $S$, such that for each nonzero ideal $I \subseteq R$, its extension $I^{\varepsilon}=S I S$ in $S$ is equal to $S$. Then $R$ is strongly prime and there exists a unique centred homomorphism $\rho: Q(R) \rightarrow S$, extending the given embedding, and sending the extended centroid $F=Z(Q(R))$ of the ring $R$ into $Z(S)$ (see [11], Theorems 2 and 5). This generalizes Amitsur's result proved for simple rings $S$ (see [1], Theorem 18). Particularly this universal property shows that the simple ring $Q(R)$ is a minimal centred extension satisfying (7) of Theorem 2.1.

Let $\phi: R \rightarrow S$ be a centred homomorphism of rings. Then for all $a, b \in$ $R$, the left and right multiplications $l_{a}, r_{b} \in M(R)$ canonically extend to $l_{\phi a}, r_{\phi b} \in M(S)$. If $\sum_{k} l_{a_{k}} r_{b_{k}}=0$ in $M(R)$, this means that $\sum_{k} a_{k} x b_{k}=0$ for all $x \in R$, we obtain $\sum_{k} \phi a_{k} \phi x \phi b_{k}=0$ in $S$. By assumption, $S$ is a 
centred extension of $R$, so each element $s \in S$ can be expressed in the form $s=\sum_{i} \phi x_{i} \delta_{i}$, where $x_{i} \in R, \delta_{i} \in Z_{S}$. So $\sum_{k} l_{\phi a_{k}} r_{\phi b_{k}}$ annihilates $s$, because the $\delta_{i}$ commute with elements from $\phi R$. Thus, sending $\lambda=\sum_{k} l_{a_{k}} r_{b_{k}} \in M(R)$ to $\sum_{k} l_{\phi a_{k}} r_{\phi b_{k}} \in M(S)$, we obtain a ring homomorphism $\phi^{\prime}: M(R) \rightarrow M(S)$. This homomorphism is centred because $M(S)$ as an $M(R)$-module is generated by the elements $l_{u} r_{v}, u, v \in Z_{S}$, which commute with all $l_{a} r_{b} \in M(R)$.

If $\phi$ is a monomorphism and $\lambda^{\prime}=\phi^{\prime} \lambda=0$ for some $\lambda \in M(R)$, then for any $x \in R, \lambda^{\prime} \phi x=0$, implying $\lambda x=0$ so $\lambda=0$ showing that $\phi^{\prime}$ is also a monomorphism. Gathering all this we obtain:

Lemma 2.3. Each centred homomorphism of rings $\phi: R \rightarrow S$ induces a canonical centred homomorphism $\phi^{\prime}: M(R) \rightarrow M(S)$. If $\phi$ is a monomorphism, then $\phi^{\prime}$ is also a monomorphism.

Now we are in a position to describe the multiplication rings of strongly prime rings.

Theorem 2.4. A ring $R$ is strongly prime if and only if its multiplication ring $M(R)$ is strongly prime. In this case their extended centroids are canonically isomorphic, and the central closure $Q(M(R)) \cong Q \otimes_{F} Q^{\circ}$, where $Q=Q(R)$ and $F=F(R)$.

Proof. Let $M(R)$ be a strongly prime ring, so $M(R)$ centrally embeds into the simple ring $Q(M(R))$. The map $R \hookrightarrow M(R)$, sending $a \in R$ to the left multiplication $l_{a} \in M(R)$, is a centred monomorphism, because $l_{a}$ commutes with all right multiplications $r_{b}, b \in R$. By (6) of Theorem 2.1, $R$ is strongly prime. By Amitsur's theorem we obtain centred embeddings of the central closures $Q(R) \subseteq Q(M(R))$ and extended centroids $F(R) \subseteq F(M(R))$.

If $R$ is strongly prime, we have a canonical centred monomorphism $\phi: R \rightarrow$ $Q$, where $Q$ is a simple ring. Then, by Lemma 2.3, $M(R)$ can be centrally embedded into $M(Q(R))$. We have already noticed that for the simple ring $Q$ with centre $F$, the multiplication ring $M(Q)$ is a simple ring $K=Q \otimes_{F} Q^{\circ}$. So $M(R)$ is strongly prime by (6) of Theorem 2.1 .

By general properties of central simple algebras, $Z(K)=F$. Also $K$ is generated by $F$ and the elements $a \otimes b^{\circ}, a, b \in R$. But these elements belong to $M(R)$. So we have $K=M(R) F$, because $Q(R)=R F$.

From Amitsur's theorem we obtain centred embeddings

$$
M(R) \subseteq Q(M(R)) \subseteq Q \otimes_{F} Q^{\circ}=K,
$$

with $F(M(R))=Z(Q(M(R)) \subseteq Z(K)=F(R)$. We already have proved that $F(R) \subseteq F(M(R))$ for the strongly prime ring $M(R)$. Thus $F(M(R))=F(R)$, and $Q(M(R))=M(R) F(M(R))=M(R) F(R)=K$. 
Theorem 2.5. Let $R$ be a strongly prime ring. If a ring $S$ is Morita equivalent to $R$, then $S$ is strongly prime and the extended centroids of $R$ and $S$ are isomorphic.

Proof. If $R$ is strongly prime, the matrix ring $M_{n}(R)$ centrally embeds into a simple $\operatorname{ring} M_{n}(Q(R))$ and is strongly prime. Also $M_{n}(Q(R))=M_{n}(R) F$, and $Z\left(M_{n}(Q(R))\right)=F$, where $F$ is the extended centroid of the ring $R$. We have $S \cong e M_{n}(R) e$, for some $n \in \mathbb{N}$, and some idempotent $e \in M_{n}(R)$. Thus

$$
e M_{n}(R) e \subseteq e M_{n}(Q(R)) e=e M_{n}(R) F e=e M_{n}(R) e e F e,
$$

because $F$ is the centre of $M_{n}(Q(R))$. But $e M_{n}(Q(R)) e$ is a simple ring whose centre $e F e$ is isomorphic to the field $F$.

Recall that a ring $R$ is semiprime if it does not contain any nonzero nilpotent ideals. It is well known that in a semiprime ring left and right annihilators of an ideal coincide, so we can speak about ideals with zero annihilators. It is also clear that an ideal of a semiprime ring $R$ is essential as an $M(R)$-submodule if and only if it has zero annihilator.

A finite set $A=\left\{a_{1}, \ldots, a_{n}\right\} \subseteq R$ is called an insulator, if

$$
A n n_{M(R)}\left\{a_{1}, \ldots, a_{n}\right\} \subseteq A n n_{M(R)}\left\{1_{R}\right\},
$$

i.e., if $\lambda a_{1}=\ldots=\lambda a_{n}=0$, implies $\lambda 1=0$.

The set $\operatorname{In}(R)$ of insulators of $R$ is evidently closed under multiplication.

In a semiprime ring $R$, insulators can be characterised in terms of the central closure $Q(R)$ and extended centroid $F(R)$. Indeed, using Theorem 32.3 in [19], we obtain:

Proposition 2.6. In any semiprime ring $R$, a finite subset $A=\left\{a_{1}, \ldots, a_{n}\right\}$ is an insulator if and only if $1 \in A F$, i.e. if

$$
a_{1} \varphi_{1}+\cdots+a_{n} \varphi_{n}=1,
$$

for suitable $\varphi_{k}$ from the extended centroid $F$ of $R$.

Denote by $\mathcal{F}$ the set of right ideals in $R$ containing an insulator. Analogously we define the set $\mathcal{F}^{\prime}$ as left ideals of $R$ containing an insulator.

If $R$ is commutative, any ideal generated by elements of an insulator is dense. It will follow from the proof of Proposition 2.7 below that in any commutative ring $\mathcal{F}$ is a Gabriel filter.

We remind that a semiprime ring $R$ is called strongly semiprime if for each essential ideal $I, R \in \sigma_{M(R)}[I]$ (see $[19,34.3]$ ). It easily follows from the definitions that $R$ is strongly semiprime if and only if each essential ideal contains an insulator. Clearly each strongly prime ring is strongly semiprime. 
Proposition 2.7. If $R$ is a strongly semiprime ring, then $\mathcal{F}$ and $\mathcal{F}^{\prime}$ are symmetric Gabriel filters. Corresponding left and right localizations form a biradical in the sense of Jategaonkar, i.e., corresponding torsion submodules in $R / A$ coincide for each ideal $A \subseteq R$.

Proof. First we prove that $\mathcal{F}$ is symmetric - this means by definition that each right ideal $U \in \mathcal{F}$ contains an ideal which belongs to $\mathcal{F}$.

By Proposition 2.6, UF $\ni 1$ and we have an expression

$$
a_{1} \varphi_{1}+\ldots+a_{n} \varphi_{n}=1, \text { with } a_{k} \in U, \varphi_{k} \in F \text {. }
$$

All $\varphi_{k}$ can be represented as $M(R)$-homomorphisms $\varphi_{k}: I_{k} \rightarrow R$, where $I_{k}$ are ideals with zero annihilators in $R$. Evidently the ideal $I=\cap_{k} I_{k} \in \mathcal{F}$, because it is essential and so contains an insulator. We show that $I \subseteq U$. Indeed, we have $x=\sum_{k} a_{k} \varphi_{k} x$, for all $x \in I$. But $\varphi_{k} x \in R$, because $x \in I_{k}$, for all $1 \leq k \leq n$. Thus $I \subseteq U$ and $\mathcal{F}$ is a symmetric set of right ideals.

Now we going to verify the axioms of Gabriel filters for $\mathcal{F}$.

First we show that for each $r \in R$ and $U \in \mathcal{F}$,

$$
(U: r)=\{x \in R \quad r x \in U\} \in \mathcal{F} .
$$

For $U \in \mathcal{F}$ we have already observed that it contains some ideal $I$ from the $\mathcal{F}$. Thus $(U: r) \supseteq I$ for all $r \in R$, so $(U: r) \in \mathcal{F}$ for all $r \in R$.

Now consider a right ideal $V$ and some $U \in \mathcal{F}$, such that $(V: u) \in \mathcal{F}$, for all $u \in U$. We must show that $V \in \mathcal{F}$, i.e., that $V$ contains an insulator.

Choose any insulator $A=\left\{a_{1}, \ldots, a_{n}\right\} \subseteq U$. For each $a_{k}$ we have insulators $B_{k}=\left\{b_{k 1}, \ldots, b_{k m}\right\} \subseteq\left(V: a_{k}\right)$, and $a_{k} b_{k l} \in V, \quad 1 \leq k \leq n, 1 \leq l \leq m$. Now $1 \in B_{k} F$ for all $1 \leq k \leq n$, because $R$ is semiprime. So $a_{k} B_{k} F \ni a_{k}$, and the set of elements $\left\{a_{k} b_{k l}\right\}=\bigcup_{k} a_{k} B_{k} \subseteq V$ is an insulator because $1 \in A F$.

Thus we proved that $\mathcal{F}$ is a symmetric Gabriel filter, with a basis consisting of finitely generated right ideals. Moreover, $U Q=Q$ for each $U \in \mathcal{F}$.

The proof for $\mathcal{F}^{\prime}$ is analogous.

Let $A \subseteq R$ be an ideal. An element $\bar{x} \in R / A, x \in R$, is torsion for $\mathcal{F}$ if and only if $\bar{x} I=0$, or equivalently, $x I \subseteq A$, for some essential ideal $I \subseteq R$. Let the elements $\left\{i_{1}, \ldots, i_{n}\right\}$ form an insulator in $I$. Then $x i_{k}=a_{k} \in A$ and $x=\sum_{k} a_{k} \varphi_{k}$, for suitable $\varphi_{k} \in F$. All $\varphi_{k}$ are defined on some essential ideal $J \subseteq R$, so we obtain that $J x \subseteq A$. This means that $\bar{x} \in R / A$ is a torsion element for $\mathcal{F}^{\prime}$. So localizations corresponding to the Gabriel filters $\mathcal{F}$ and $\mathcal{F}^{\prime}$ form a biradical. 
Theorem 2.8. Let $R$ be a strongly semiprime ring. Then the canonical map

$$
\phi: Q(R) \otimes_{R} Q(R) \rightarrow Q(R)
$$

is an isomorphism, and $Q(R)$ is flat as a left and a right $R$-module.

Proof. Consider an element $x=\sum_{k} r_{k} u_{k} \otimes v_{k}$ in the kernel of $\phi$, where $r_{k} \in$ $R, \quad u_{k}, v_{k} \in F$, i.e. $\sum_{k} r_{k} u_{k} v_{k}=0$.

All $v_{k}$ can be represented as $M(R)$-homomorphisms $v_{k}: I_{k} \rightarrow R$, where $I_{k}$ are essential ideals. Then the ideal $I=\bigcap_{k} I_{k} \neq 0$ is also essential, thus contains an insulator $\left\{i_{1}, \ldots i_{n}\right\}$. Then $\sum_{l} i_{l} \varphi_{l}=1$, for some $\varphi_{l} \in F$. We have

$$
x=\sum_{k} r_{k} u_{k} \otimes\left(\sum_{l} i_{l} \varphi_{l}\right) v_{k}=\sum_{l}\left(\sum_{k} r_{k} u_{k} v_{k}\right) i_{l} \otimes \varphi_{l}=0,
$$

because all $v_{k} i_{l} \in R$.

To prove that $Q(R)$ is flat as a right $R$-module consider a left ideal $L \subseteq R$, and the canonical homomorphism

$$
\phi^{\prime}: Q(R) \otimes_{R} L \rightarrow Q(R) .
$$

Let $a=\sum_{k} u_{k} \otimes r_{k}$ be in the kernel of $\phi^{\prime}$, where $u_{k} \in F$ and $r_{k} \in L$, i.e. $\sum_{k} u_{k} r_{k}=0$ in $Q(R)$. All $u_{k}$ are defined as $M(R)$-homomorphisms on some essential ideal $J$, so we have $\sum_{l} j_{l} \psi_{l}=1$, with suitable $j_{l} \in J, \psi_{l} \in F$. Thus

$$
a=\sum_{k} u_{k}\left(\sum_{l} j_{l} \psi_{l}\right) \otimes r_{k}=\sum_{l} \psi_{l} \otimes \sum_{k}\left(u_{k} j_{l}\right) r_{k}=0
$$

because all $u_{k} j_{l} \in R$, and $\sum_{k}\left(u_{k} j_{l}\right) r_{k}=j_{l} \sum_{k} u_{k} r_{k}=0$, for all $l$. This shows that $Q(R)$ is flat as a right $R$-module.

Left flatness can be proved analogously.

A ring homomorphism $\phi: R \rightarrow S$, for which the canonical map $S \otimes_{R} S \rightarrow S$ is an isomorphism and which induces the structure of a right (left) flat $R$ module is called a right (left) flat epimorphism.

The theorem proved shows that for a strongly semiprime ring the canonical embedding $R \rightarrow Q(R)$ is a right and left flat epimorphism.

By a theorem of Popescu-Spircu (see [16], Ch. XI, Theorem 2.1), for each right flat epimorphism $\phi: R \rightarrow S$, the set of right ideals

$$
\mathcal{F}=\left\{U_{R} \subseteq R \quad \phi(U) S=S\right\}
$$

is a Gabriel filter and $S$ is canonically isomorphic to the quotient ring $Q_{\mathcal{F}}(R)$.

By Proposition 3.4 in [16], Ch. XI, for any right flat epimorphism $\phi: R \rightarrow S$, $M \otimes_{R} S \cong Q_{\mathcal{F}}(M)$, for each $M \in M o d-R$, i.e. the localization, associated with a flat epimorphism, is perfect. 
Applying the Popescu-Spircu Theorem to the embedding $R \rightarrow Q(R)$ for a strongly semiprime ring $R$, and the characterisation of Gabriel filters in Proposition 2.7 and Theorem 2.8, we obtain:

Theorem 2.9. Let $R$ be a strongly semiprime ring. Then

(1) $Q(R) \otimes_{R} Q(R) \cong Q(R)$,

(2) $Q(R)$ is flat as left and right $R$-module,

(3) the sets $\mathcal{F}$ and $\mathcal{F}^{\prime}$ are symmetric Gabriel filters, the corresponding localizations are perfect,

(4) the central closure $Q(R)$ is canonically isomorphic to the quotient ring of $R$ with respect to $\mathcal{F}$ and $\mathcal{F}^{\prime}$.

It is worth noticing that the following lemma (also) implies one of the equivalent conditions of the Popescu-Spircu Theorem and from this the statements of Theorem 2.9 can be regained.

Lemma 2.10. Let $R$ be a strongly semiprime ring. Then for every $q \in Q(R)$, there exist elements $i_{1}, \ldots, i_{n} \in R$ and $\psi_{1}, \ldots, \psi_{n} \in F$, such that $q i_{k}, i_{k} q \in R$, and $\sum_{k} i_{k} \psi_{k}=1$.

Proof. Let $q=r_{1} \varphi_{1}+\ldots+r_{m} \varphi_{m}, r_{k} \in R, \varphi_{k} \in F$.

We have already noticed that all $\varphi_{k}$ can be represented as $M(R)$-homomorphisms $\varphi_{k}: I_{k} \rightarrow R$, where $I_{k}$ and $I=\bigcap_{k} I_{k}$ are in $\mathcal{F}$, so $I$ contains an insulator. Thus we have $1=\sum_{k} i_{k} \psi_{k}$, for some $i_{k} \in I$ and $\psi_{k} \in F$. But $\varphi_{k} i \in R$, for all $i \in I$, so $q i_{k}, i_{k} q \in I$, for $1 \leq k \leq n$.

\section{Strongly PRIME IDEALS}

An ideal $\mathfrak{p} \subset R$ is called strongly prime if the factor ring $R / \mathfrak{p}$ is a strongly prime ring.

We can adapt Theorem 2.1 for equivalent characterizations of strongly prime ideals. From (5) of this theorem we obtain the following:

Proposition 3.1. An ideal $\mathfrak{p} \subset R$ is strongly prime if and only if for each $a \notin \mathfrak{p}$, there exist elements $a_{1}, \ldots, a_{n} \in(a), n=n(a)$, such that for each $\lambda \in M(R)$ with $\lambda 1 \notin \mathfrak{p}$, at least one of elements $\lambda a_{k} \notin \mathfrak{p}$.

Clearly, maximal ideals are strongly prime. It is well known that in PI rings each prime ideal is strongly prime. Of course, any strongly prime ideal is prime by (3) of Theorem 2.1. Since not each prime ring has a simple central closure, prime ideals are not necessarily strongly prime. Using standard arguments we easily obtain from Theorem 2.5 that strongly prime ideals are preserved under Morita equivalences. If $\phi: R \rightarrow S$ is a centred homomorphism of rings, and 
$\mathfrak{q} \subset S$ is a strongly prime ideal, we easily obtain from(6) of Theorem 2.1 that $\mathfrak{p}=\phi^{-1} \mathfrak{q}$ is a strongly prime ideal in $R$.

The intersection of all strongly prime ideals of the $\operatorname{ring} R$ we call the strongly prime radical and denote it by $\operatorname{sr}(R)$. We give a characterization of the strongly prime radical of the ring. Let $R\left[X_{1}, \ldots X_{n}\right]$ be a polynomial ring over the ring $R$ with commuting or noncommuting indeterminates.

Theorem 3.2. $a \in \operatorname{sr}(R)$ if and only if for any $a_{1}, \ldots, a_{n} \in(a)$, the ideal in $R\left[X_{1}, \ldots, X_{n}\right]$, generated by the polynomial $a_{1} X_{1}+\ldots+a_{n} X_{n}-1$ contains 1 .

Proof. If some polynomial $a_{1} X_{1}+\ldots+a_{n} X_{n}-1$ generates a proper ideal in $R\left[X_{1}, \ldots, X_{n}\right]$, we can take a maximal ideal $\mathcal{M} \subset R\left[X_{1}, \ldots, X_{n}\right]$ containing this polynomial. Evidently $a \notin \mathcal{M}$. So we have the centred homomorphims $\phi: R \rightarrow R\left[X_{1}, \ldots, X_{n}\right] / \mathcal{M}$ with $\phi a \neq 0$ and $\phi^{-1} \mathcal{M}$ is a strongly prime ideal in $R$ not containing $a$. This implies $a \notin \operatorname{sr}(R)$.

Now assume $a \notin \operatorname{sr}(R)$. Then $a \notin \mathfrak{p}$ for some strongly prime ideal $\mathfrak{p} \subset R$, and therefore $(\bar{a})^{\varepsilon}=Q(R / \mathfrak{p})$, yielding an expression

$$
\bar{a}_{1} u_{1}+\cdots+\bar{a}_{n} u_{n}=1 \text { in } Q(R / \mathfrak{p}),
$$

with $\bar{a}_{1}, \ldots, \bar{a}_{n} \in(\bar{a}), \quad u_{1}, \ldots, u_{n} \in F(R / \mathfrak{p})$.

So the polynomial $a_{1} X_{1}+\ldots+a_{n} X_{n}-1$ is in the kernel of the homomorphism $R\left[X_{1}, \ldots, X_{n}\right] \rightarrow Q(R / \mathfrak{p})$, which sends $X_{k}$ to the $u_{k}$, for $1 \leq k \leq n$. Thus the ideal generated by this polynomial is proper.

This theorem is an analogue of the well-known fact that an element $a$ of any commutative ring $R$ is nilpotent if and only if the polynomial $a X-1$ is invertible in $R[X]$.

Since each maximal ideal is strongly prime, the strongly prime radical of the ring is contained in the Brown-McCoy radical.

Theorem 3.3. The strongly prime radical $\operatorname{sr}(R)$ of $R$ contains the Levitzki radical $L(R)$.

Proof. Recall that the Levitzki radical is the largest locally nilpotent ideal of the ring. If some element $a \in L(R)$ is not in the strongly prime radical, we have an expression

$$
\text { (*) } \quad \bar{a}_{1} u_{1}+\ldots+\bar{a}_{n} u_{n}=1 \text { in } Q(R / \mathfrak{p}),
$$

with $a_{1}, \ldots, a_{n} \in(a), \quad u_{1}, \ldots, u_{n} \in F(R / \mathfrak{p})$, for some strongly prime ideal $\mathfrak{p} \subset R$. Because $A=\left\{a_{1}, \ldots, a_{n}\right\}$ is in $L(R)$, there exists $m \in \mathbb{N}$ such that all products $a_{k_{1}} \ldots a_{k_{m}}$ with $a_{k_{l}} \in A$ are zero. Then the $m$-th power of the expression $(*)$ would give a contradiction. 
Problem. It would be interesting to know if - or under which conditions the upper nilradical of the ring is contained in $\operatorname{sr}(R)$.

Recall that a subset $A \subseteq R$ of a ring is an m-system if $1 \in A$ and for each $a, b \in A$, arb $\in A$ for some $r \in R$. Two main properties of the $m$-systems are well known: a complement of a prime ideal is an $m$-system, and each ideal maximal with respect to being disjoint with $A$ is prime.

Now we introduce a variation of this notion and characterize strongly prime ideals in terms of these sets.

We call a subset $\mathcal{S} \subseteq R$ strongly multiplicative, or sm-set, if $1 \in \mathcal{S}$ and for any $a \in \mathcal{S}$ there exist elements $a_{1}, \ldots, a_{n} \in(a), n=n(a)$, such that for each $\lambda \in M(R)$ with $\lambda 1 \in \mathcal{S}$, we have $\lambda a_{k} \in \mathcal{S}$, for some $1 \leq k \leq n$.

Proposition 3.4. If $\mathfrak{p} \subset R$ is a strongly prime ideal, its complement in $R$ is a strongly multiplicative set.

Proof. The assertion is just another form of Proposition 3.1.

Other examples of $s m$-sets are obtained from any ideal $I \subset R$ :

$$
\mathcal{S}=\{1+i \mid i \in I\} \subseteq R \text { is an sm-set. }
$$

Indeed, for each $a=1+i, i \in I$ take $n=1$ and $a_{1}=a$. If $\lambda 1=1+j, j \in I$, then $\lambda a=1+j+\lambda i \in \mathcal{S}$, showing that $\mathcal{S}$ is strongly multiplicative.

Theorem 3.5. Let $\mathcal{S} \subset R$ be a strongly multiplicative set not containing 0 . Then each ideal $\mathfrak{p} \subset R$, maximal with respect to $\mathfrak{p} \cap \mathcal{S}=\emptyset$, is strongly prime.

Proof. Let $x \notin \mathfrak{p}$. Then $p+\mu_{0} x=a \in \mathcal{S}$, for some $p \in \mathfrak{p}$ and $\mu_{0} \in M(R)$. Let

$$
a_{k}=\lambda_{k} a=\lambda_{k} p+\lambda_{k} \mu_{0} x \in(a), 1 \leq k \leq n,
$$

be elements corresponding to $a$ in the definition of $s m$-sets. Let $\lambda 1 \notin \mathfrak{p}$. Then $q+\nu_{0} \lambda 1=\left(l_{q}+\nu_{0} \lambda\right) 1=\lambda^{\prime} 1 \in \mathcal{S}$, for some $q \in \mathfrak{p}$, where $l_{q} \in M(R)$ is the left multiplication by $q$. Then for some $k, \lambda^{\prime} a_{k} \in \mathcal{S}$ thus not in $\mathfrak{p}$. So we have

$$
\lambda^{\prime} a_{k}=\left(l_{q}+\nu_{0} \lambda\right)\left(\lambda_{k} p+\lambda_{k} \mu_{0} x\right)=q a_{k}+\nu_{0} \lambda \lambda_{k} p+\nu_{0} \lambda \lambda_{k} \mu_{0} x \notin \mathfrak{p} .
$$

But $q a_{k}$ and $\nu_{0} \lambda \lambda_{k} p$ are in $\mathfrak{p}$, so $\lambda \lambda_{k} \mu_{0} x \notin \mathfrak{p}$. Thus, for each $x \notin \mathfrak{p}$, there exist a finite set of elements $x_{k}=\lambda_{k} \mu_{0} x \in(x)$, such that for each $\lambda \in M(R)$ with $\lambda 1 \notin \mathfrak{p}$, at least one of the elements $\lambda x_{k} \notin \mathfrak{p}$. By Proposition 3.1, the ideal $\mathfrak{p}$ is strongly prime.

Let $\mathcal{S} \subset R$ be a strongly multiplicative set. Similar to the commutative case, we define the set

$$
\mathcal{S}^{\prime}=\{u \in R \mid(u) \cap \mathcal{S} \neq \emptyset\}
$$

and call it the saturation of $\mathcal{S}$. We call $\mathcal{S}$ saturated if $\mathcal{S}^{\prime}=\mathcal{S}$. 
Denote by $H$ the union of all strongly prime ideals $\mathfrak{p} \subset R$ disjoint with $\mathcal{S}$. We have shown that $H \neq \emptyset$ when $0 \notin \mathcal{S}$.

Proposition 3.6. Let $\mathcal{S}$ be a strongly multiplicative set. Then $\mathcal{S}^{\prime}$ is also strongly multiplicative and $\mathcal{S}^{\prime}=R \backslash H$.

Proof. This is shown similarly to the commutative case.

Corollary 3.7. For a commutative ring saturated strongly multiplicative sets are the usual saturated multiplicative sets.

\section{REFERENCES}

[1] Amitsur, S.A., On rings of quotients, Symp. Mathematica 8(1972), 149-164.

[2] Artin, M., On Azumaya algebras and finite dimensional representations of rings, J. Algebra 11(1969), 532-563.

[3] Bahturin, Y.A., Basic Structures of Modern Algebra, Moscow, Nauka 1990.

[4] Beachy, J., Some aspects of noncommutative localization, Noncommutative Ring Theory, LNM, vol 545, Springer-Verlag, 1975, 2-31.

[5] Beidar, K.I., Martindale 3rd, W.S., Mikhalev, A.V., Rings with Generalized Identities, Pure and Applied Math. 196, Marcel Dekker Inc., New York 1996.

[6] Beidar, K.I., Wisbauer, R., Strongly and properly semiprime rings and modules, Ring Theory, ed. by Jain-Rizvi e.a., World Scientific, Singapore, 1993, 58-95.

[7] Goldman, O., Elements of noncommutative arithmetic I, J. Algebra 35(1975), 308-341.

[8] Delale, J.-P., Sur le spectre d'un anneau non commutatif, Thèse, Universite Paris Sud, Centre d'Orsay, 1974.

[9] Handelman, D., Lawrence, J., Strongly prime rings, Trans. Amer. Math. Soc. 211(1975), 209-223

[10] Jara, P., Verhaege, P., Verschoren, A., On the left spectrum of a ring, Comm Algebra 22(8), (1994), 2983-3002.

[11] Kaučikas, A., On centred and integral homomorphisms, Lith. Math.J. 37(3), 1997, 264268.

[12] Passman, D., Computing the symmetric ring of quotients. J. Algebra, 105(1987), 417448.

[13] Popescu, N., Spircu, T., Quelques observations sur les épimorphismes plats (à gauche) d'anneaux, J. Algebra 16(1970), 40-59.

[14] Rosenberg, A.L., Noncommutative Algebraic Geometry and Representations of Quantized Algebras, Kluwer, Dordrecht, 1995.

[15] Van Oystaeyen, F., Verschoren, A., Noncommutative Algebraic Geometry, LNM 887, Springer Verlag, Berlin 1981.

[16] Stenström, B., Rings of Quotients, Springer Verlag, Berlin 1981.

[17] Wisbauer, R., Localizations of modules and the central closure of rings, Comm. Algebra 9(1981), 1455-1493.

[18] Wisbauer, R., On prime modules and rings, Comm. Algebra 11(1983), 2249-2265.

[19] Wisbauer, R., Modules and Algebras: Bimodule Structure and Group Action on Algebras, Pitman Monographs and Surveys in Pure and Applied Mathematics, vol 81, Addison Wesley, Longman 1996. 
Faculty of Mathematics, Vilnius Pedagogical University, Studentu 39, VilNIUS, 2034, LiTHUANIA

E-mail address: al.kauchikas@vpu.lt

Institute of Mathematics, Heinrich-Heine-University, 40225 Düsseldorf, GerMANY

E-mail address: wisbauer@math.uni-duesseldorf.de 